Engagierte Nachwuchs-Führungskräfte

\section{Pflegemanagement-Award 2016 verliehen}

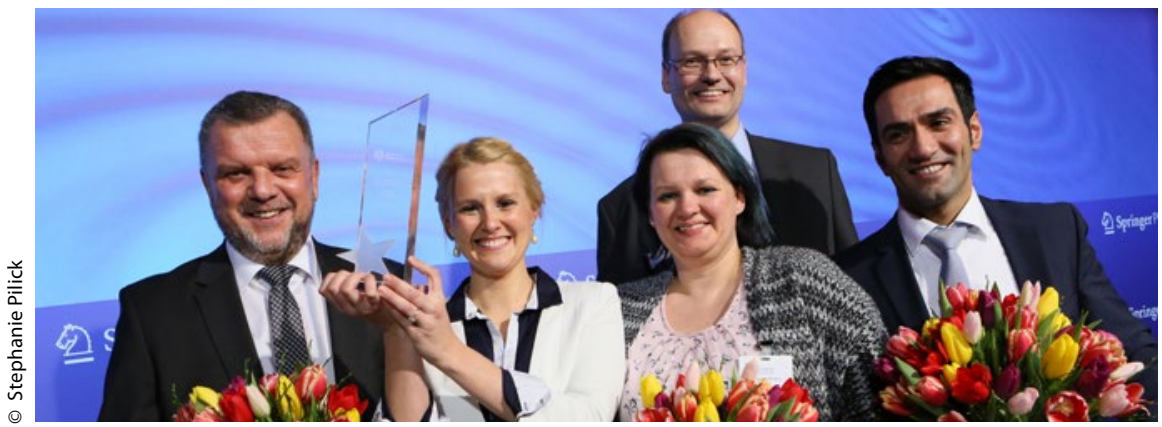

Laudatoren und Preisträger - Peter Bechtel, Sarah Behling, Doreen Hoffmann, Rüdiger Herbold und Mustafa Atas (v.I.n.r.).

— Der Pflegemanagement-Award für Nachwuchsführungskräfte wurde während einer Abendveranstaltung anlässlich des von Springer Pflege veranstalteten Kongresses Pflege 2016 überreicht. Bei dem Preis, der bereits zum fünften Mal verliehen wurde, handelt es sich um eine Initiative des Bundesverbands Pflegemanagement mit Unterstützung von Springer Pflege. Hauptsponsor war erneut ZeQ, eines der führenden Beratungsunternehmen in der Gesundheitswirtschaft.

Das Grußwort zur Preisverleihung sprach auch in diesem Jahr der Bevollmächtigte der Bundesregierung für Patienten und Pflege, Staatssekretär Karl-Josef Laumann:
„Der Pflegemanagement-Award ist ein starkes Zeichen der Würdigung für das besondere Engagement der jungen Nachwuchsführungskräfte". Mit dem Pflegemanagement-Award werden Nachwuchsführungskräfte ausgezeichnet, die sich mit Engagement, Kreativität sowie sozialer und fachlicher Kompetenz für die Stärkung der Position der Profession Pflege einsetzen und dabei das Wohl des Patienten stets im Zentrum ihres Handels sehen. Der Preisverleihung vorausgegangen war ein zweistufiges Auswahlverfahren, bei dem aus den zahlreichen erstklassigen Nominierungen zunächst die besten zehn und schließlich drei Preisträger ausgewählt wurden.
Der erste Platz ging an Sarah Behling, stellvertretende Stationsleitung am St. Christophorus-Krankenhaus in Werne. "Sarah Behling steht für eine neue Generation von Führungskräften in der Pflege, wie sie die Profession Pflege dringend braucht, um den Versorgungsprozess auf Augenhöhe mitgestalten zu können. Mit einem hohen $\mathrm{Maß}$ an Fachkompetenz überzeugt sie durch Offenheit und Transparenz auf allen Ebenen", so Peter Bechtel, Vorstandsvorsitzender des Bundesverbands Pflegemanagement.

Mustafa Atas, Stationspflegeleitung am Vivantes-Klinikum Neukölln kam auf Platz zwei. „Durch seine aktive Teilnahme am Versorgungsprozess gelingt es ihm in erstaunlichem Maß, Pflegepraxis mit Management-Theorie in Einklang zum Wohl der Patienten zu bringen. Eine herausragende Pflege-Persönlichkeit", kommentierte Rüdiger Herbold, Vorstand von ZeQ, die Preisverteilung. Den dritten Platz erreichte Doreen Hoffmann, Stationsleiterin am Klinikum Bamberg. „Ihre Überzeugung, dass mehr Versorgungseffizienz zu mehr Patienten- und Mitarbeiterzufriedenheit führt, steht für eine gelebte Kombination aus fachlicher und sozialer Kompetenz", so Staatsekretär Karl-Josef Laumann.

www.gesundheitskongresse.de
Online-Befragung

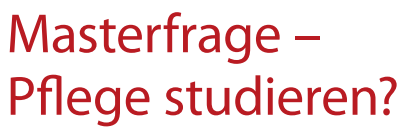

— In Vorbereitung eines berufsbegleiten den Masterstudienganges für die Pflege untersucht das Projekt PRAWINA an der Evangelischen Hochschule Dresden die individuelle Sicht auf Professionalisierung und Qualifizierungsbedarf. Bis zum 17. April 2016 können Leitungspersonen, Pflegefachkräfte, Studierende, Dozenten und Referenten im Bereich Pflege und Gesundheitsversorgung unter dem Link ww2.unipark.de/ uc/pflege-master/ an der Onlinebefragung teilnehmen.

www.ehs-dresden.de
Lernwelten 2016

\section{Kultursensibel pflegen - Call for Abstracts}

— Lehrende und Angehörige der Gesundheitsfachberufe müssen in der Bildungsbeziehungsweise Arbeitspraxis zunehmend mit einer Vielfalt von Kulturen, Lebenswelten und Wirklichkeiten der Akteure umgehen. Der 16. Lernweltenkongress, der vom 1.-3. September in Wien stattfindet, thematisiert "Kultursensibilität" in der Pflege- und Gesundheitsaus-, -fort und -weiterbildung und in gesundheitsbezogenen Studiengängen. Lehrende, Wissenschaftler/-innen, Hochschullehrer/-innen und Studierende aus dem Gesundheits-, Pflege- und Sozialbereich können noch bis zum 30. März ihre Abstracts einreichen. Die wissenschaftlichen Beiträge, Erfahrungsberichte, BestPractice-Beispiele und studentischen Arbeiten sollten sich mit folgenden Perspektiven beschäftigen: Kultursensibel lernen, lehren, und arbeiten. Wie können Migranten für die Gesundheitsberufe gewonnen werden? Welche pädagogischen Anforderungen ergeben sich daraus? Welche Unterstützung ist dafür von welcher Seite notwendig?

www.lernwelten.info

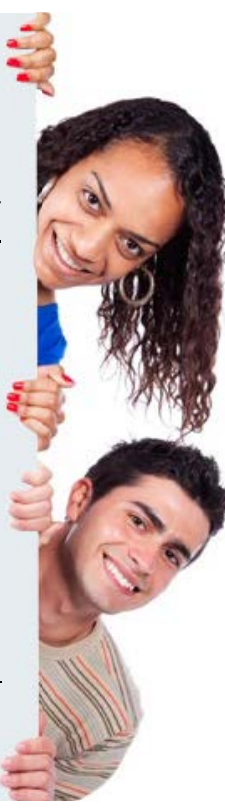

